\title{
GA Optimized S-Band Quadrafilar Antenna with the Lowest Back Radiation for a Communication Satellite
}

\author{
Sungtek Kahng ${ }^{1} \cdot$ Joongpyo $\mathrm{Kim}^{2}$
}

\begin{abstract}
In this paper, a quadrafilar antenna is designed to have a lower profile as an essential part of the size reduction technique and lower back radiation(i.e. higher forward radiation) for the S-band telemetry/telecommand(TM/TC) function of a communication satellite. Particularly, to meet the challenging requirements on the higher isolation between the TM/TC antennas and simultaneously a smaller size, the lowest back radiation and lowest cross-polarization, the optimal physical dimensions of the quadrafilar antenna are found by using the Genetic Algorithm(GA). To prove the validity of the proposed antenna design, its $3 \mathrm{D}$ electromagnetic analysis and measured results are compared, showing good agreement.
\end{abstract}

Key words: Quadrafilar Antenna, Optimization, Genetic Algorithm.

\section{I . Introduction}

Showing its unique features compared to other antenna-using applications, the satellite is required to be equipped with a variety of antennas for different multiple functions, but calls for strict and complex specifications for high quality performance, including the environment qualification. The waveguide horn antennas represent the antennas used for satellite communication.

However, helical antennas are also adopted ${ }^{[1]}$. The helical antennas are easy to design and handle. But this is true for easy-to-realize cases occurring in ground wireless communication, and it is quite complicating and difficult to meet the satellite telemetry and telecommand functions, which, usually drive the antenna design in order to have the lowest interference possible between the telemetry and telecommand antennas with low 'backradiation'(or high 'forward radiation') and a low crosspolarization level. This stems from the fact that a helical antenna has a relatively smaller number of design parameters $^{[1]}$.

When it comes to the application to telemetry and telecommand, the circular polarization(CP) is recommended; one type of $\mathrm{CP}$ antenna(i.e. right-handed $\mathrm{CP}$ or RHCP) is assigned to the telemetry and the other type of CP antenna(i.e. left-handed CP or LHCP) is for the telecommand to maximize the cross-polarization performance and isolation between the TM and TC functions. They are separated from each other by 180 degrees in order to minimize the interference between them. However, assuming the RHCP antenna is positioned in the top region with respect to the horizontal bisection-line of a satellite and the LHCP is in the bottom area, the cross-polarization component of the RHCP turns into the LHCP, which is the back radiation from the top region to the bottom region and will interfere with the LHCP of the original antenna for the bottom. To avoid this, Kilgus suggested a method to lower the back radiation and maximize the forward radiation by the quadrafilar helical antenna which shows a very flat forward pattern in either RHCP or $\mathrm{LHCP}^{[2] \sim[4]}$, but it usually requires several turns which will end up with the increment of the antenna height.

In this paper, we use the GA(Genetic Algorithm $)^{[5]}$ ${ }^{[10]}$ to find the optimal values of the quadrafilar antenna design parameters for a smaller structure, not limited to the Kilgus' design guideline. Through the GA optimization process, we find a way to lower the back radiation and to have flatness in terms of the RHCP antenna residing on the top area of the satellite, and reveal the optimized performance.

\section{Basis for the Proposed Design}

\section{2-1 Quadrafilar Antenna to be Used for the Commu- nication Satellite}

The quadrafilar antenna is placed on the top or bottom area of a satellite, working in an S-band.

The quadrafilar antenna takes the compound of four helical antennae with a sequential $90^{\circ}$ phase difference between the two neighboring helixes. This antenna is

Manuscript received October 28, 2009 ; revised December 14, 2009. (ID No. 20091028-046J)

${ }^{1}$ Dept. of Information \& Telecommunication. Engineering, University of Incheon, Incheon, Korea.

${ }^{2}$ Korea Aerospace Research Institute, Daejon, Korea. 


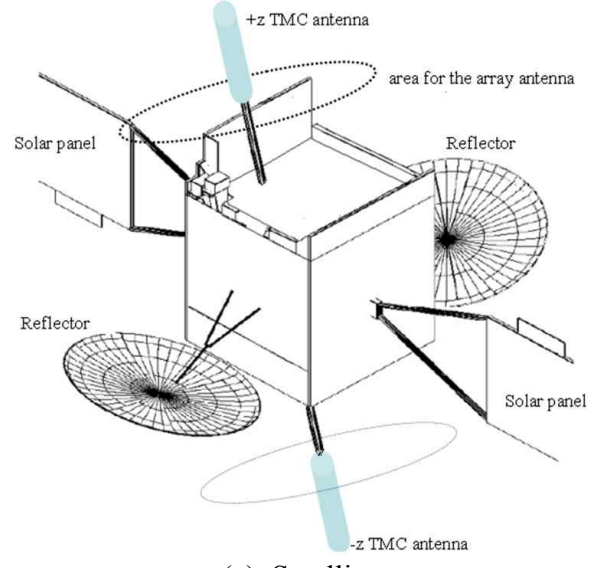

(a) Satellite

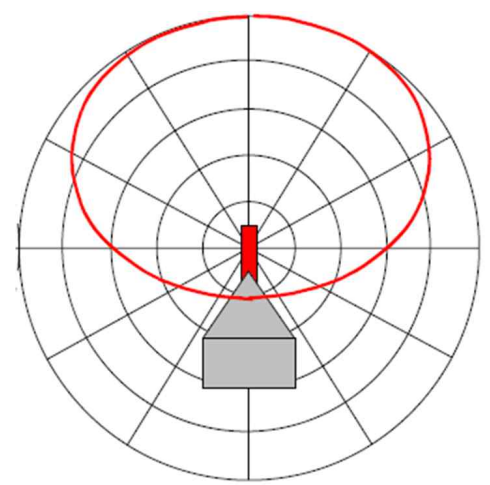

(b) Forward radiation required for TM/TC functions

Fig. 1. Sketch of a communication satellite with multiple antennas mounted on its surface, including wire antennas for the purpose of TM/TC(Telemetry/Telecommand).

assigned to the telemetry function in the top area of a satellite and will be designed to have the RHCP propagation. To avoid the interference from the metal plates of the top region and the degrading interference to the antenna on the other side, this antenna should have the lowest and weakest back radiation possible. Here, the number of the turns, the radius and the height of the antenna are designated as the physical dimensions for the design. To predict the electromagnetic field of the antenna according to the geometrical, boundary and material conditions, the Method of Moment(MoM) solver of the FEKO is used. On a side note, in terms of the degree of freedom in design, due to the limited number of the physically changeable parameters, it is a tough call in satisfying the challenging requirements. This is why we need an optimization algorithm, such as a stochastic search, in the following section.

\section{2-2 Genetic Algorithm}

The GA is employed to get the design parameters'

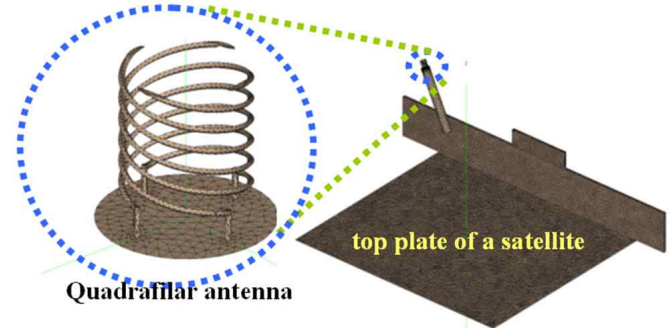

(a) 3D quadrafilar antenna mounted on the vehicle

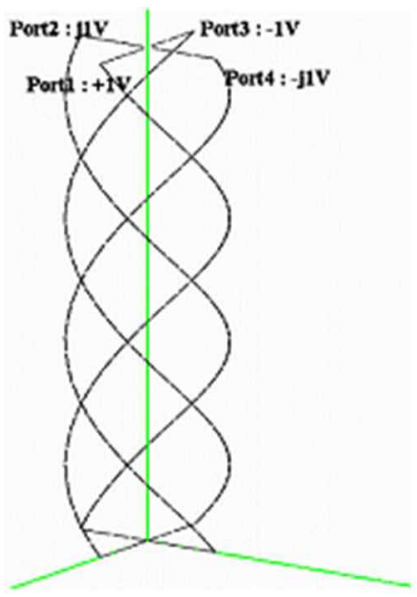

(b) Quadrafilar antenna for the proposed design

Fig. 2. Illustration of the quadrafilar antenna on the top area of a satellite.

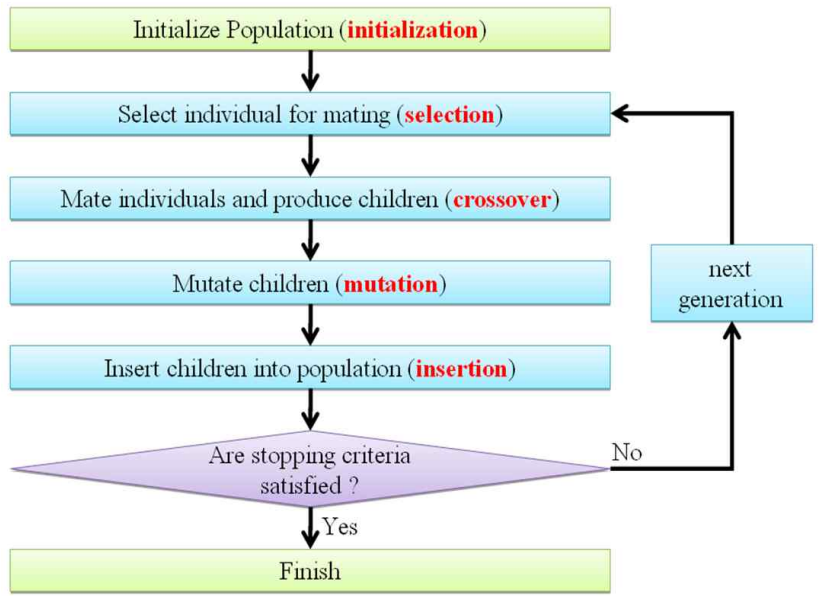

Fig. 3. The optimization process of Genetic Algorithm.

values that are optimal in producing the required performances $^{[5] \sim[10]}$. Below, we briefly address the GA it stochastically searches the global minimum in the variable space of the cost function, while performing selection, mating, crossover, mutation, and reproduction.

As always, this optimization starts with defining the cost function as

$$
\operatorname{Cost}_{1}=\sum_{q=1}^{N a} n_{q}\left|P\left(\Theta_{q}\right)-P\left(\Theta_{q-1}\right)\right|^{N e}
$$




$$
\operatorname{Cost}_{2}=\sum_{p=1}^{N \underline{g}} \xi_{p}\left|P\left(\Theta_{p}\right)\left(P_{R e f}\right)\right|^{N e}
$$

where the cost function 1 as eqn. (1), with weight $\eta_{q}$, considering the 1 st portion in the total cost, is the error between the two immediate points of $N_{q}$ samples along the elevation angle as the RHCP E-field, and used to make the flat RHCP pattern. At the same time, with another weight $\xi_{p}$ and order $N_{e}$, the cost function 2 as eqn. (2) is the inverse of the summation of the differences between the field's magnitudes $P\left(\Theta_{p}\right)$ at $N_{p}$ angular points and the level $P_{\text {Ref }}$ of $-25 \mathrm{~dB}$ (or empirically at highest $-20 \mathrm{~dB}$ ) as the reference threshold points for the cross-polarization or back radiation, i.e. LHCP. Once again, eqn (2) is intended to maximize the gap between the $-25 \mathrm{~dB}$ (or empirically, at highest $-20 \mathrm{~dB}$ ) and back radiation level. These two cost functions make up one total cost function. The genes (or binary bits) of the GA are generated as random variables for the design parameters $N_{\text {turn }}$ (number of turns), $R_{\text {qudra }}$ (radius), and $h_{\text {qudra }}$ (height), each of which has $N_{b i t}$ binary bits. Each of the $N_{\text {pop }}$ (number of population) individuals comprizes 5 $N_{u}$ genes, where $N_{u}$ is the number of genes. Afterwards, the population undergoes Selection, Crossover with rate $P_{C r}$, and Mutation with rate $P_{m}$ over $N_{\text {genr }}$ generations, with Elitism specifically for this work.

\section{Design, Realization and Validation}

The working frequency is $2.09 \mathrm{GHz}$ and the total gain is set at $3 \mathrm{dBi}$. In line with this, the following table shows the detailed specifications on the quadrafilar antenna design.

Although most of the specifications above are dependent on this particular satellite development, the gain, bandwidth and polarization look very similar to other helical-type of antennas. However, what makes the difference between the present design and most antenna designs is the isolation and cross-polarization level observed at the broadside angle, i.e. $\Theta=0^{\circ}$. Even if neither the

Table 1. Specifications on the quadrafilar antenna performance.

\begin{tabular}{|l|c|}
\hline \multicolumn{1}{|c|}{ Item } & Specification \\
\hline Center frequency & $2.09 \mathrm{GHz}$ \\
\hline Bandwidth (reflection $<-10 \mathrm{~dB})$ & $0.7 \mathrm{GHz}$ \\
\hline Gain & $\geq 3 \mathrm{dBi}$ \\
\hline Type of Co-polarization & RHCP \\
\hline Half power beam-width $(=\mathrm{HPBW})$ & $\approx 60$ degrees \\
\hline Cross-polarization level at the broadside & $\mathrm{LHCP}=-55 \mathrm{~dB}$ \\
\hline Isolation at the broadside & $\geq 55 \mathrm{~dB}$ \\
\hline
\end{tabular}

gain nor the HPBW seems very large, it does not matter in this case, where the TM antenna on the top and TC antenna beneath the bottom of the satellite should not interfere with each other, and this is determined by the highest isolation $(\geq 55 \mathrm{~dB})$ and lowest cross-polarization level. This is the forward-only radiation or lowest back radiation possible from each of the TM and TC antennas.

Note we define the radius, the height, etc as the genes (=variables or design parameters) of the GA's individuals(=sets of parameters). Prior to the presentation of the GA optimization results, the optimization is carried out with cost function 1 to have flat forward radiation(Sampled RHCP E-field over $-60^{\circ}<\theta<60^{\circ}$ should have extremely small variation) and cost function 2 to have the lowest back radiation (Sampled LHCP E-field over $-60^{\circ}<\theta<60^{\circ}$ should be lower than $-20 \mathrm{~dB}$ or -25 dB) separately. To run the GA optimization code, we have given $N_{b i t}=5, N_{p o p}=200, N_{g e n r}=100, P_{C r}=0.5$, and $P_{m}$

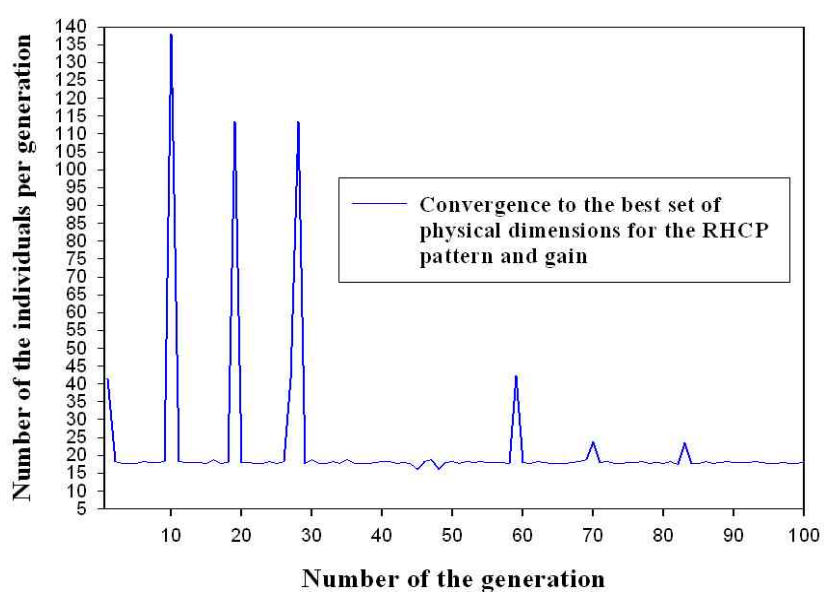

(a) Best number of individuals per generation

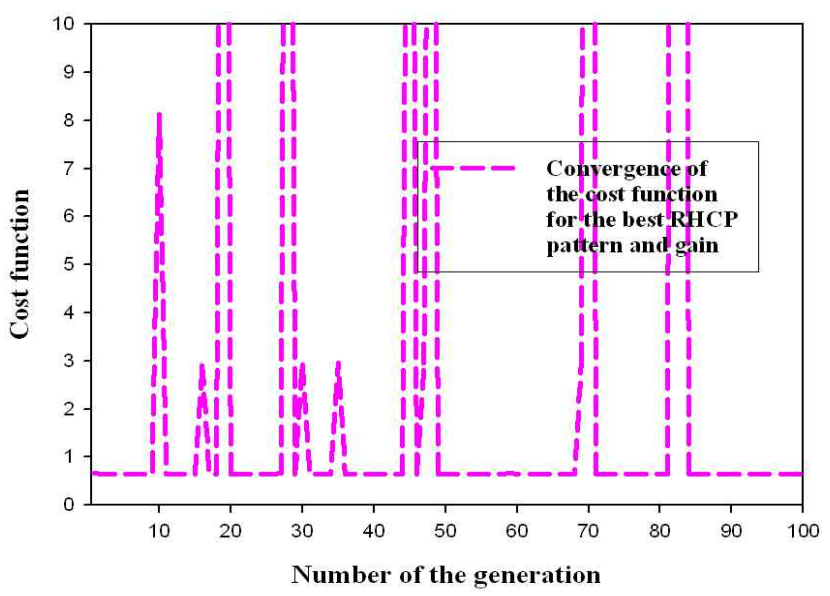

(b) Cost function

Fig. 4. Searching the optimal set of physical dimensions and minimizing the cost function to flatten the field radiation in the forward direction. 
$=0.1$ as the inputs. Also, $\eta_{\mathrm{p}}$ and $\xi_{p}$ are set $1 / N_{p}$ and $1 / N_{q}$ for equal weighting over samples, where $N_{p}$ and $N_{q}$ are 20. Besides, we have set $N_{e}=1$. First, we choose ' $P_{\text {Ref }}$ $=-20 \mathrm{~dB}$ condition for cost function $2^{\prime}$ and check convergence to find the optimal set of physical dimensions.

Fig. 4 shows how the optimal design parameter set is being found, and that the cost function is settling down. Actually, the average of the individuals or parameters per generation can be plotted, but its traces will cross one another and get tangled and confuse observers. Consequently, here we monitor the number(as the ID) of the parameter set(individual) best in each generation and plot it. Though the best few parameter sets, as the generation progresses, are very similar in terms of the cost function as well as the design parameters, the parameter sets' IDs as integers can be the same, close, or very far apart. Thus, Fig. 4 has some peaks, since the best sets of different generations have similar parameter values but different IDs. Fig. 4(a) tells us that numbers 22 and 17 are the best parameter sets among 200 individuals in generation 80 and generations 90 , or 100 , respectively. In Fig. 4(b), at first sight, the convergence of the cost function is not good, there being peaks right before the end of the generation. As a result, it provides us the following acceptable performance from the final best parameter set.

To check the forward-only radiation or lowest backradiation as the key to the high isolation between the TM and TC antennas, it is worthwhile to note the difference between the RHCP and LHCP levels at $\theta=0^{\circ}$. It amounts to around $73 \mathrm{~dB}$ right at the center. It has acceptable isolation. However, the cross-polarization level goes beyond $-20 \mathrm{~dB}$ at $\theta=+60^{\circ}$. Therefore, we desire to fix this problem by putting the LHCP under $-20 \mathrm{~dB}$.

Second, we choose ${ }^{\prime} P_{R e f}=-25 \mathrm{~dB}$ condition for cost

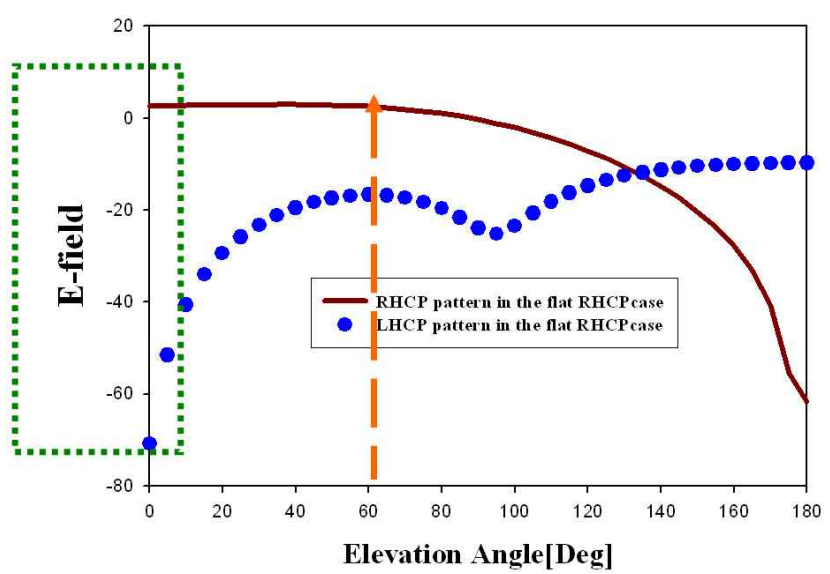

Fig. 5. The RHCP(solid line) shows the flat forward radiation, but LHCP(dotted line) is acceptable but not completely optimized.

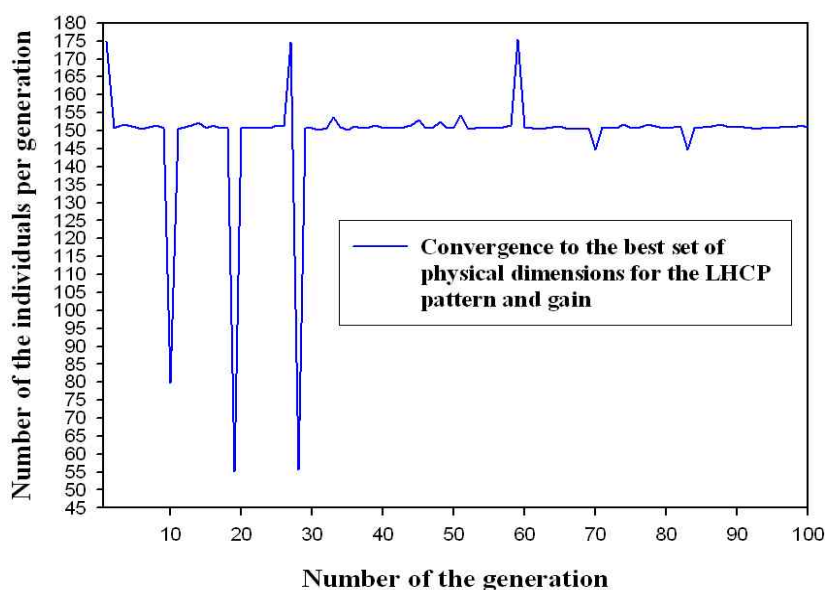

(a) Best individual per generation

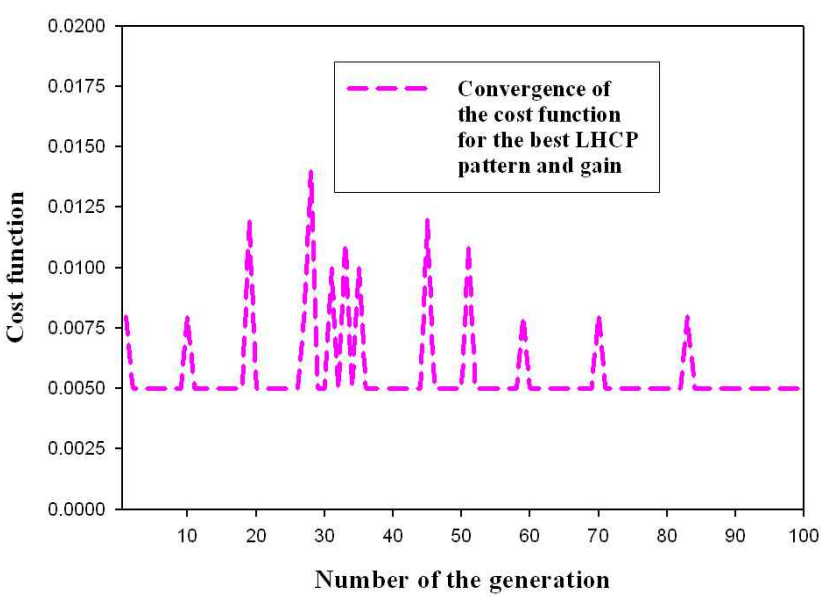

(b) Cost function

Fig. 6. Searching the optimal set of physical dimensions and minimizing the cost function to lower the LHCP.

function $2^{\prime}$ and watch the convergence for the optimal design to lower the LHCP comfortably below $-20 \mathrm{~dB}$, particularly at $\theta=+60^{\circ}$.

According to Fig. 6(a), while the generation progresses, the best parameter set becomes 150 . With the new condition for cost function 2 , the cost becomes much smaller than before to the order of 3 , as shown in Fig. 6(b). Hence, we obtain the performance of the RHCP and LHCP as follows.

With Fig. 7 as the second design of the forward-only radiation or lowest back-radiation essential to the high isolation between the TM and TC antennas, it is worth noting the difference between the RHCP and LHCP levels at $\theta=0^{\circ}$. It is seen to be about $82 \mathrm{~dB}$. It turns out to be another enormous isolation. Also, the cross-polarization level is under $-20 \mathrm{~dB}$ over the HPBW. Through the above process of the work, we finalize the design. The optimized antenna has got physical dimensions, the radius of $11.5 \mathrm{~mm}$, and the height of $65 \mathrm{~mm}$ in a half- 


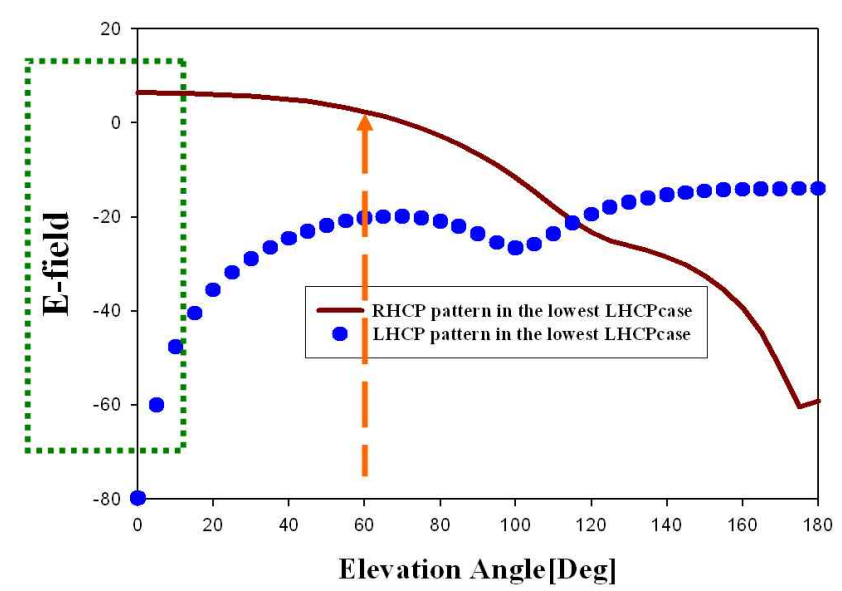

Fig. 7. The LHCP(dotted) and the RHCP(solid) improved by more steps of the optimization.

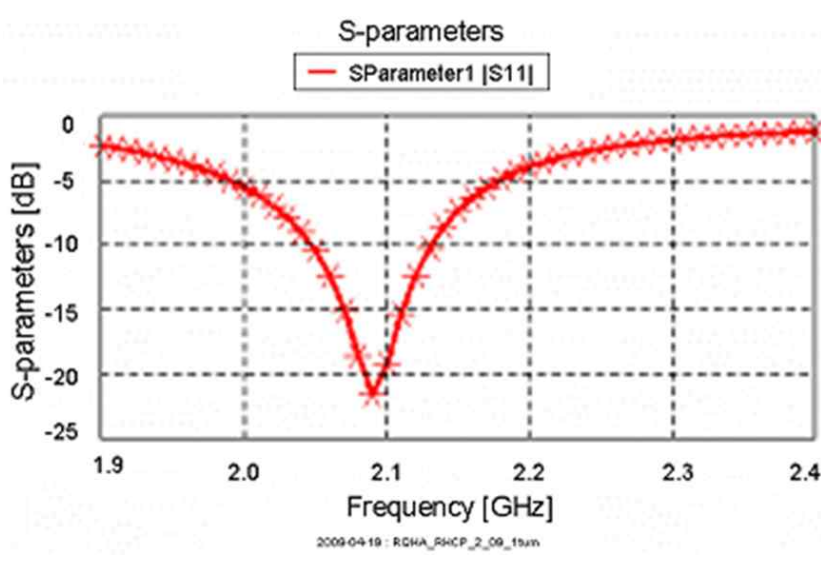

Fig. 8. Return loss of the proposed antenna.

turn geometry that are smaller than one-turn case with the radius of $11.5 \mathrm{~mm}$ and the height of $130 \mathrm{~mm}$. Using this physical structure, the return loss of the optimized antenna is as follows.

As is targeted, the frequency band below the -10 dB-reflection coefficient has a center frequency of 2.09 $\mathrm{GHz}$ and bandwidth of $700 \mathrm{MHz}$. Finally, the E-field pattern is analyzed with respect to the proposed quadrafilar antenna.

The optimized antenna's pattern performance is addressed with the 3D electric field pattern and the comparison between co-polarization and cross-polarization levels as a check to the isolation. Both results show the wanted gain $(3.3 \mathrm{dBi})$, the maximum radiation in the forward direction, and almost zero back radiation at $\theta=180^{\circ}$. In particular, the predicted field pattern agrees well with the measurement. Despite the small discrepancy in Fig. 9(b), we can see the analyzed and measured patterns with low cross-polarization and maximized isolation for the TM and TC antennas. This proposed antenna has

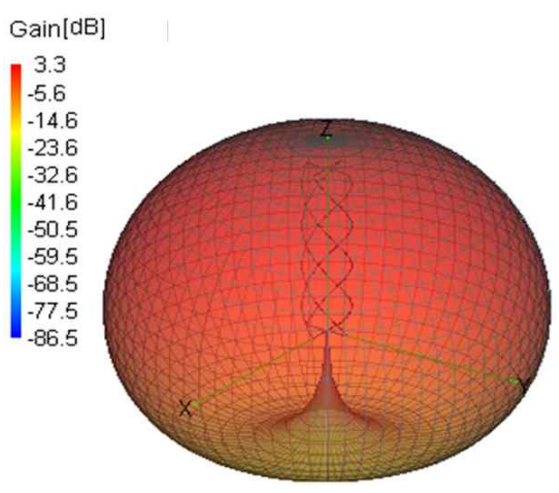

(a) 3D electric field pattern

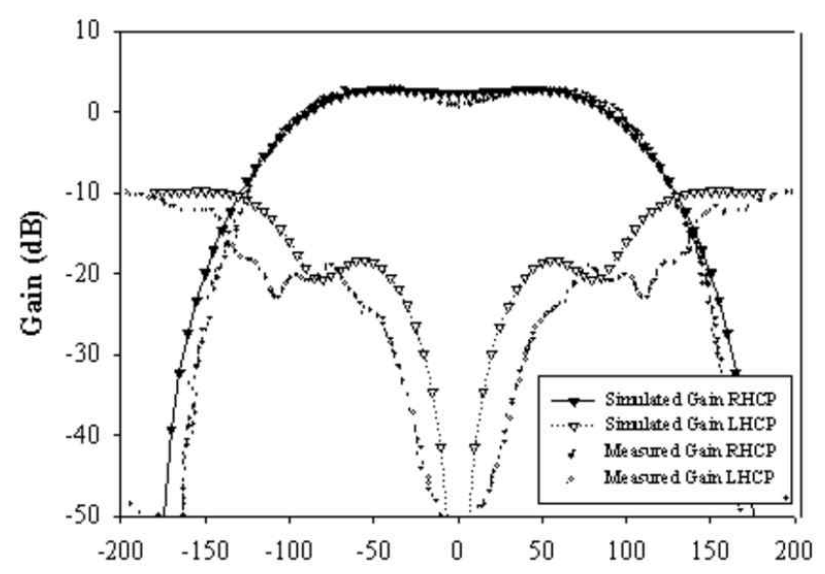

(b) Co-pol(RHCP) and cross-pol(LHCP) levels

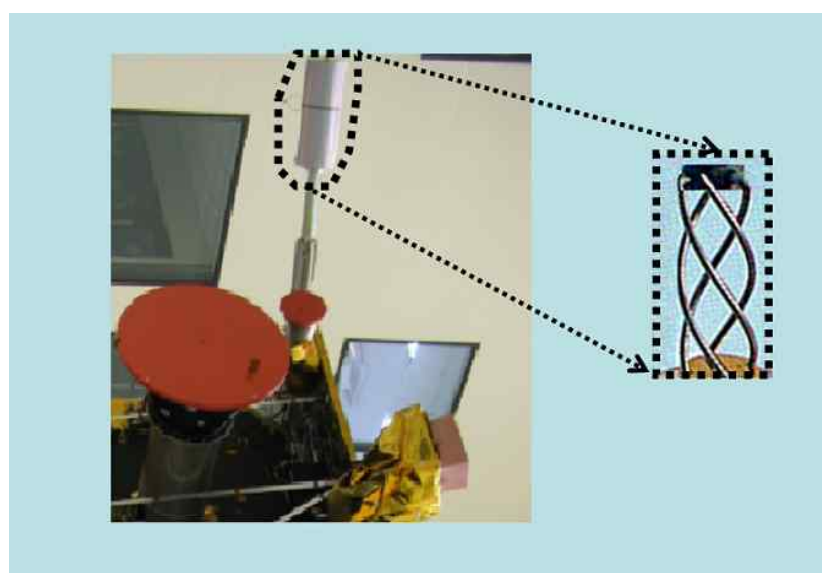

(c) Fabricated and mounted antenna

Fig. 9. Field patterns and photo of the realized antenna.

been fabricated and is shown in Fig. 9(C).

\section{Conclusions}

We presented the design of the highest RHCP radiation and lowest LHCP(forward-only) radiation using a low-profiled S-band quadrafilar antenna on the satellite. The challenging requirements of the size and the tough specifications on the lowest back-radiation have been 
met by the use of the GA optimization approach. The design has been validated by comparing the predicted and measured performances.

\section{References}

[1] S. E. J. D. Kraus, R. J. Marhefka, Antennas for All Applications, N.Y., USA: McGraw Hill, 2002.

[2] C. Kilgus, "Multi-element, fractional turn helices", IEEE Trans. On AP, vol. 16, pp. 499-500, 21572158, Jul. 1968.

[3] M. Sekelja, J. Jurica, and Z. Blazevic, "Designing and testing the quadrafilar gelical antenna", Software, International Conference on Telecommunications \& Computer Networks, pp. 55-59, Sep. 2009.

[4] L. Hadj Abderrahmane, M. Benyettou, and M. N. Sweeting, "An S-band antenna system used for communication on earth observation microsatellite", IEEE, Aerospace Conference, 2006.

[5] Sungtek Kahng, "The rectangular power-bus with slits GA-optimized to damp resonances", IEEE Trans. on Antenna \& Propagation, vol. 55, no. 6, pp. 18921895, Jun. 2007.

[6] Sungtek Kahng, "GA-optimized decoupling capaci-

\section{Sungtek Kahng}

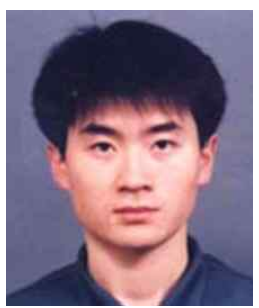

was with Hanyang University in Seoul, Korea and there he received the Ph.D. degree in electronics and communication engineering in 2000, with the specialty in radio science and engineering. From year 2000 to early 2004, he worked for the Electronics and Telecommunication Research Institute, where he worked on numerical electromagnetic characterization of and developed the $\mathrm{RF} /$ microwave/millimeter passive components for satellites. Since March 2004, he has joined the department of Information and Telecommunication engineering at University of Incheon that he has continued studying analysis and advanced design methods of microwave components and antennas. Along with the above, he is accredited to be in the Science \& Engineering of Marquis Who's Who in the World and holds several patents concerning metamaterials, RF components and EMC solutions as well. tors damping the rectangular power-bus' cavity-mode resonances", IEEE Microwave and Wireless Components Letters, vol. 16, no. 6, pp. 375-377, Jun. 2006.

[7] Sungtek Kahng, "Genetic algorithm-based complex images for magnetic dipoles in parallel plates", IET Electronics Letters, vol. 39, no. 8, pp. 667-668, Apr. 2003.

[8] Sungtek Kahng, "Rotation-free transformation of the coupling matrix with genetic algorithm-error minimizing pertaining transfer functions", Journal of The Korean Institute of Electromagnetic Engineering And Science, vol. 4, no. 3, pp. 102-106, Sep. 2004.

[9] Sungtek Kahng, "An efficient filter design via optimized rational-function fitting, without similarity transformation", Journal of The Korean Institute of Electromagnetic Engineering and Science, vol. 6, no. 3, pp. 155-159, Sep. 2006.

[10] Sungtek Kahng, Jeongho Ju, "The design of a dielectric rod antenna using genetic algorithm optimization for vessel's collision avoidance applications", Journal of The Korean Institute of Electromagnetic Engineering and Science, vol. 7, no. 3, pp. 129133, Sep. 2007.

\section{Joongpyo Kim}

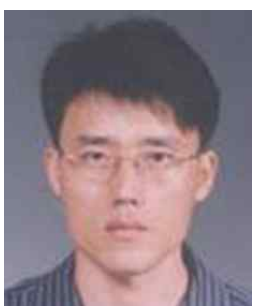

received B.E., M.E. and Ph.D degrees from Kyungpook National University in 1991, 1993, and 2000, respectively, all in Electronic Engineering. Since March 2000, he has worked in the Korea Aerospace Research Institute, Daejon, Korea, developing RF equipment for satellites, being involved in various projects like COMPSAT,

COMS, etc. 